\title{
Trajectory Generation and Control of a 9 axis CNC Micromachining Center
}

\author{
A. Yuen, Y. Altintas (1)* \\ Manufacturing Automation Laboratory, Department of Mechanical Engineering, The University of British Columbia, Vancouver, BC, V6T 1Z4, Canada
}

A novel 9-axis micro-machine is presented. The machine has three linear motor driven major axes and a magnetically levitated rotary table with six degrees of freedom. The table can rotate freely with pitch, roll, and yaw and three linear short stroke motions. The major drives bring the tool to the vicinity of target location while the magnetically levitated table positions the machine accurately. All nine drives are controlled simultaneously by considering the redundant kinematics of the machine without saturating the stroke limits. Simulation and experiments show the generated reference commands do not violate the limits of the feed drives, and is sufficiently accurate.

Micro machining, Multi-axis, Redundant, Kinematics, Trajectory

\section{Introduction}

There has been a growing interest in the development of multi-axis micromachining centers with precision positioning capability due to the miniaturization of engineering components [1] [2]. Sample applications include micro-impeller, micro electromechanical devices, surface texturing and micro-dies and molds [3].

The conventional machines with serial kinematic configuration lead to accumulated inertia on the main carriage which reduce the bandwidth hence the tracking accuracy. The frictional contacts at the drives lead to poor positioning accuracy, and the use of air bearings to reduce the friction can be costly and disturbance stiffness may suffer. It is preferred to have a machine with multiple degrees of freedom (DOF) to access various part features with a minimum number of set ups, and reduce the frictional contacts as much as possible to achieve precision positioning at high feed speeds.

Kinematically redundant machine tools have been introduced to improve the accessible work area and enhance the precision positioning with excess drives [4]. Takeuchi et. al [5] suggested frictionless aerostatic drives to improve the positioning accuracy, and added a $6^{\text {th }}$ axis to a 5 -axis machine to micro-mill miniature parts with complex shapes [6]. Lu and Usman presented a frictionless, magnetically levitated high speed table with 6 DOF with a large planar workspace [7]. The same magnetic levitation principle has been used in designing a rotary table with 6 DOF [8], and integrated to a 3 axis micro-machine to create a 9 DOF micro-machine presented in this paper. The three axis Cartesian gantry machine has a granite structure for thermal stability. Although multi-axis machine tools exist in the field of micro-manufacturing, very few have redundant degrees of freedom as the configuration presented here. Choi et. al [9] presents a similar configuration, however it's rotation about the $\mathrm{C}$-axis is limited, restricting its possible positioning capabilities. The combined use of the rotary table presented in [8], and its increased positioning capabilities, particularly in the $\mathrm{C}$-axis, make the presented novel 9DOF machine capable of machining miniature features, free form surfaces, and surface textures with a variety of micro geometries. Though it is possible to manufacture complex parts with a 5-axis machine, 4 additional axes provide capabilities such as geometric and tracking error compensation, or increasing the bandwidth of a redundant axes. The fundamental challenges are how to generate 5 - axis tool path trajectory with smooth velocity, acceleration and jerk using 4 extra, redundant drives without saturating any of the actuators' stroke, velocity, acceleration, and jerk limits, which are challenges that have not been fully addressed. Though works exist for planning trajectories with toolpath limits [10] and configurations without redundancy [11] [12], feed planning for redundant configurations has yet to be addressed.

This paper presents a novel 9 degree of freedom machine tool including the trajectory generation algorithm and control strategy that allows simultaneous motion on all drives without saturating them. The proposed algorithms are experimentally demonstrated on a spatial helical path.

\section{Overview of the 9-axis Micro-machine Tool}

The 9-axis micro-machine tool consists of a main, three axis Cartesian machine body carrying a magnetically levitated table with 6 DOF as shown in Figure 1. The main drives are magnetically driven linear motors with $\mathrm{x}, \mathrm{y}$, and $\mathrm{z}$ peak forces of $364 \mathrm{~N}, 580 \mathrm{~N}$, and $458 \mathrm{~N}$, and continuous forces of $91 \mathrm{~N}, 145 \mathrm{~N}$, and $73 \mathrm{~N}$ respectively. Each axis is capable of moving along the Cartesian axes $\mathrm{x}, \mathrm{y}, \mathrm{z}$ with a stroke of $100 \mathrm{~mm}$ with an interpolator supported encoder resolution of $40 \mathrm{~nm}$. Air bearing supported direct driven Fischer spindle with 200,000 $\mathrm{rev} / \mathrm{min}$ is mounted on the $\mathrm{z}$ axis of the machine.

The rotary table is magnetically driven as shown in [8], where the mover consists of a circular Halbach magnet array and the stator is a circular printed circuit board (PCB). Actuation is achieved by passing current through copper coils in the PCB, which creates an opposing magnetic force to the magnets in the mover. The rotary table has a stroke limit of $100 \mu \mathrm{m}$ in the $\mathrm{x}, \mathrm{y}$, and $\mathrm{z}$ axes, and 0.5 degrees in the $\mathrm{A}$ and $\mathrm{B}$ axes, and unlimited rotation in the $\mathrm{C}$ axis. The translational axes have a resolution of $20 \mathrm{~nm}$ while the rotational axes have a resolution of $0.6 \mu \mathrm{rad}$. Since the table is non-contact, problems associated with traditional rotary tables, such as friction and backlash, are eliminated [8].

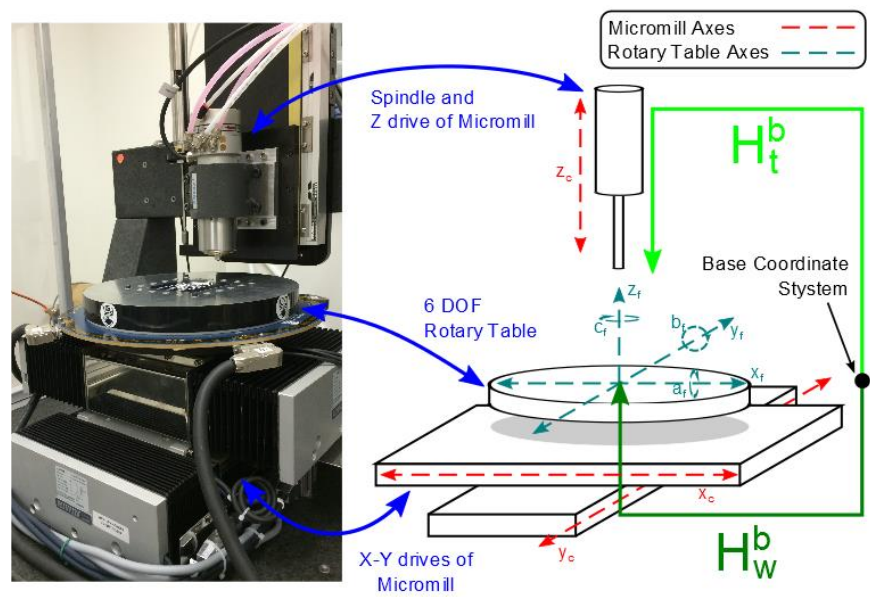

Figure 1. UBC MAL's 9-axis micromaching center and the kinematic chains

The magnetic actuator is fastened to the top of the X-Y axes of the micro-mill as shown in Figure 1. The rotary table is configured so that the translational axes of the rotary table are parallel with the translational axes of micro-mill. With 3 DOF from the micro-mill and $6 \mathrm{DOF}$ from the rotary table, the micromachining center has 9 DOF. The micro-machine is controlled by a single dSpace DS1103 realtime computer. The real-time computer provides three PWM channels and three encoder channels to the micro-mill for current commands and position feedback, respectively. For the rotary table, 4 encoder channels, four analog-to-digital channels, and 8 digital-to-analog channels are required. The 4 encoder channels measure 4 translational locations of the rotary table, providing solutions for the $\mathrm{x}, \mathrm{y}$, and $\mathrm{C}$ position of the rotary table. The 4 analog-to-digital channels interface 
with 4 capacitance probes that measure the height of 4 different locations of the rotary table, thus solving for the $\mathrm{z}, \mathrm{A}$, and $\mathrm{B}$, position of the rotary table. The 8 digital-to-analog channels are needed to control the 4, three-phase linear amplifiers to actuate the rotary table. As a result, 7 encoder channels, 3 PWM channels, 8 digital-to-analog converters, and 4 analog-to-digital converters are used to control the whole machine.

Since the machine has more DOF than necessary, a trajectory generation strategy has been developed so that the generated position commands correspond to the desired tooltip position and orientation. Furthermore, given the different dynamics of the micro-mill and rotary table, control strategies have been developed for the different drives. The machine is controlled by an in-house developed, modular, open architecture CNC running on real-time MATLAB supported dSpace digital signal processing board.

\section{Trajectory Generation for the 9-axis Micro-machine Tool}

The position of a micro-end mill attached to the spindle can be represented by its Cartesian coordinates $\boldsymbol{P}=\left[P_{x} P_{y} P_{z}\right]^{T}$ and orientation $\boldsymbol{O}=\left[\begin{array}{lll}O_{i} & O_{j} & O_{k}\end{array}\right]^{T}$ vectors with respect to the workpiece. Since the micromachining center has more DOF than necessary, the relative motion between the tool and workpiece need to be delivered by simultaneously controlling 9 drive positions,

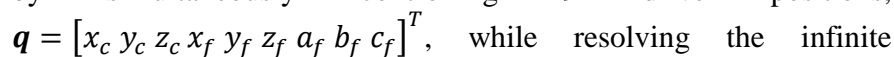
combination of drive positions possible. The subscripts $c$ and $f$ indicate axes on the micro-mill and rotary table, respectively. Furthermore, the position commands must be scheduled so the limits of the drives are not violated while traversing the desired path as fast as possible.

3.1. Forward Kinematics: Given the tool position $\left(\boldsymbol{t}_{t}^{p}\right)$ and tool orientation $\left(\boldsymbol{t}_{t}^{o}\right)$ in the tool coordinate system specified in NC tool path, the position and orientation of the tool with respect to the workpiece can be evaluated from the drive positions with the following homogenous transformation,

$$
\left[\begin{array}{ll}
\boldsymbol{P} & \boldsymbol{o} \\
1 & 0
\end{array}\right]=\boldsymbol{H}(\boldsymbol{q})\left[\begin{array}{cc}
\boldsymbol{t}_{t}^{p} & \boldsymbol{t}_{t}^{o} \\
1 & 0
\end{array}\right]
$$

where $\boldsymbol{H}(\boldsymbol{q})$ is the coordinate transformation matrix from the tool coordinate system to the workpiece coordinate system. The inverse of the coordinate transformation from the base coordinate system to the workpiece is given as:

$$
\boldsymbol{H}(\boldsymbol{q})_{w}^{b}=\left[\begin{array}{cccc}
\mathrm{c}_{b_{f}} \mathrm{c}_{c_{f}} & -\mathrm{c}_{b_{f}} \mathrm{~s}_{c_{f}} & \mathrm{~s}_{b_{f}} & x_{c}+x_{f} \\
\mathrm{c}_{a_{f}} \mathrm{~s}_{c_{f}}+ & \mathrm{c}_{a_{f}} \mathrm{c}_{c_{f}}- & & \\
\mathrm{c}_{c_{f}} \mathrm{~s}_{a_{f}} \mathrm{~s}_{b_{f}} & \mathrm{~s}_{a_{f}} \mathrm{~s}_{b_{f}} s_{c_{f}} & -\mathrm{c}_{b_{f}} \mathrm{~s}_{a_{f}} & y_{c}+y_{f} \\
\mathrm{~s}_{a_{f}} \mathrm{~s}_{c_{f}}- & \mathrm{c}_{c_{f}} \mathrm{~s}_{a_{f}}+ & & \\
\mathrm{c}_{a_{f}} \mathrm{c}_{c_{f}} \mathrm{~s}_{b_{f}} & \mathrm{c}_{a_{f}} \mathrm{~s}_{b_{f}} \mathrm{~s}_{c_{f}} & \mathrm{c}_{a_{f}} \mathrm{~s}_{b_{f}} & z_{f} \\
0 & 0 & 0 & 1
\end{array}\right]
$$

where $x_{c} y_{c} z_{c} x_{f} y_{f} z_{f} a_{f} b_{f} c_{f}$ are the drive commands of the machine. The trigonometric terms are shortened for brevity as follows: $\quad c_{a_{f}}=\cos a_{f}, \quad \mathrm{~s}_{a_{f}}=\sin a_{f}, \quad \mathrm{c}_{b_{f}}=\cos b_{f}, \quad \mathrm{~s}_{b_{f}}=\sin b_{f}$, $\mathrm{c}_{c_{f}}=\cos c_{f}$ and $\mathrm{s}_{c_{f}}=\sin c_{f}$. The transformation from the base coordinate system to the tool:

$$
\boldsymbol{H}(\boldsymbol{q})_{t}^{b}=\left[\begin{array}{cccc}
1 & 0 & 0 & 0 \\
0 & 1 & 0 & 0 \\
0 & 0 & 1 & z_{c} \\
0 & 0 & 0 & 1
\end{array}\right]
$$

where $z_{c}$ is the $\mathrm{z}$ axis command of the machine. The transformation matrix $\boldsymbol{H}(\boldsymbol{q})$ is evaluated as (Figure 1):

$$
\boldsymbol{H}(\boldsymbol{q})=\left(\boldsymbol{H}(\boldsymbol{q})_{w}^{b}\right)^{-1} \boldsymbol{H}(\boldsymbol{q})_{t}^{b}
$$

As a result, the tooltip position $\boldsymbol{P}(\boldsymbol{q})$ and tool axis orientation $\boldsymbol{O}(\boldsymbol{q})$ are expressed by the positions of all 9 drives specified in vector $(\boldsymbol{q})$.

3.2. Inverse Kinematics: The cutter location commands given in the NC program are mapped to the drives using the inverse kinematics, which are solved at the differential level with the Jacobian matrix $(J(\boldsymbol{q}))$ of the 9 axis micro-machine. The Jacobian $(J(\boldsymbol{q}))$ is obtained by taking the derivative of $\boldsymbol{P}(\boldsymbol{q})$ and $\boldsymbol{O}(\boldsymbol{q})$ from Eq. (1) with respect to the drive positions as shown:

$$
J(\boldsymbol{q})=\left[\begin{array}{ccc}
\frac{d P_{x}}{d x_{c}} & \cdots & \frac{d P_{x}}{d c_{f}} \\
\vdots & \ddots & \vdots \\
\frac{d O_{k}}{d x_{c}} & \cdots & \frac{d O_{k}}{d c_{f}}
\end{array}\right]
$$

The Jacobian maps the differential motion change of the drives to the differential change of the tool position $(d \boldsymbol{R})$ as:

$$
d \boldsymbol{R}=J(\boldsymbol{q}) d \boldsymbol{q}
$$

where $\boldsymbol{R}=[\boldsymbol{P}(s) \boldsymbol{O}(s)]^{T}$ is a vector with the desired position and orientation of the tool as a function of the displacement $(s)$ along the toolpath. The tool position $(\boldsymbol{P}(s))$ and orientation $(\boldsymbol{O}(s))$ are jerk continuous splines as described in [13]. If $d \boldsymbol{R}$ is known, then $d \boldsymbol{q}$ can be found based on the method proposed in [14]. First, the MoorePenrose pseudoinverse of the Jacobian is found as:

$$
J^{\dagger}(\boldsymbol{q})=J(\boldsymbol{q})^{T}\left(J(\boldsymbol{q}) J(\boldsymbol{q})^{T}\right)^{-1}
$$

Since the desired tool position and orientation are specified as a function of displacement, $d \boldsymbol{R} / d s$ can be found analytically. The differential of the drive positions with respect to displacement $d \boldsymbol{q} / d s$ is found from Eq. (7) as [15]:

$$
\frac{d \boldsymbol{q}}{d s}=J^{\dagger}(\boldsymbol{q}) \frac{d \boldsymbol{R}}{d s}-\alpha\left(\boldsymbol{I}-J(\boldsymbol{q})^{T} J^{\dagger}(\boldsymbol{q})^{T}\right) \nabla \varphi(\boldsymbol{q})
$$

which leads to many solutions of $d \boldsymbol{q} / d s$ because of extra redundant drives of the machine. Only one feasible solution, which gives the lowest motions of all drives to reach target position without violating the stroke limits of the micro-machine and rotary table, needs to be identified. The first term of Eq. (8), $J^{\dagger}(\boldsymbol{q})(d \boldsymbol{R} / d s)$ gives the minimum average movements of all drives to travel $d s$ [16]. The second term of Eq. (8) guarantees the avoidance of saturating the stroke limits of all drives by selecting the cost function $\varphi(\boldsymbol{q})$ as follows [15].

with the gradient:

$$
\varphi(\boldsymbol{q})=\frac{x_{c}^{2}}{x_{c, \max }^{2}}+\frac{y_{c}^{2}}{y_{c, \max }^{2}}+\cdots+\frac{c_{f}^{2}}{c_{f, \max }^{2}}
$$

$$
\nabla \varphi(\boldsymbol{q})=\left[\frac{2 x_{c}}{x_{c, \text { max }}^{2}}, \frac{2 y_{c}}{y_{c, \max }^{2}}, \ldots, \frac{2 c_{f}}{c_{f, \max }^{2}}\right]^{T}
$$

$\alpha$ is a weight factor between the lowest movements of all drives (i.e. first part of Eq. (8)) and stroke limit avoidance (i.e. second part of Eq. (8)), and must be iteratively searched to satisfy all stroke limits for all segments along the path. From Eq. (9) and Eq. (10) it can be seen that deviations from the center of the drives are penalized. The solution attempts to keep the drive positions centered without violating the stroke limits. In short, all 9 drives move to achieve a 5 axis tool path, without exceeding the short stroke limits of the 
magnetically rotary table. From the geometric derivative, $d \boldsymbol{q} / d s$, the corresponding drive positions can be found by using a numerical integration scheme such as the forward Euler integration as follows,

$$
\boldsymbol{q}[n+1]=\boldsymbol{q}[n]+\frac{d \boldsymbol{q}[n]}{d s} \Delta s
$$

where $\Delta s$ is a uniform displacement interval on the toolpath and $n$ indicates the $n^{\text {th }}$ interval of command generation. A solution for Eq. (8) and Eq. (11) will always be possible since there are no constraints on the geometric derivatives

The result of this algorithm are axes commands $\boldsymbol{q}$, scheduled at fixed toolpath displacement intervals $\Delta s$. This information can then be used with a feed optimization strategy which takes this as an input [11] [12] and plans the tangential feedrate, so the limits of the servo drives are not violated.

\section{Control of the 9 drives of the machine}

The linear motors of the 3-axis micro-mill and the magnetically actuated free floating mass of the 6-axis rotary table are controlled independently as follows:

4.1. Linear Motor Control: The open loop transfer function between the position $(X)$ and applied magnetic force $(F)$ can be modelled by the following rigid body dynamics:

$$
\frac{X}{F}=\frac{1}{M s^{2}+B s}
$$

where $M$ is the mass of the mover and $B$ is the viscous friction from the guideways with roller bearing contact. A proportional position controller cascaded with a proportional-integral velocity controller is used to control the linear drives at the initial development of the machine. The closed loop transfer function of the axis position $(X)$ is derived for a given reference position command $\left(X_{R}\right)$ as:

$$
\frac{X}{X_{R}}=\frac{K_{p} K_{v} s+K_{p} K_{v i}}{M s^{3}+\left(B+K_{v}\right) s^{2}+\left(K_{v i}+K_{p} K_{v}\right) s+K_{p} K_{v i}}
$$

where $K_{p}$ is the position controller gain, $K_{v}$ is the velocity controller gain and $K_{v i}$ is the gain of the integral velocity controller. The gains are selected so the velocity loop and position loop has a bandwidth of $60 \mathrm{~Hz}$ and $20 \mathrm{~Hz}$, respectively. The performance is further increased with a feed-forward controller to compensate for the non-linear static friction of the drives.

4.2. Rotary Table Control: The rotary table is a free floating mass, and its transfer function between the position $(X)$ and applied magnetic force $(F)$ which can be modelled as follows

$$
\frac{X}{F}=\frac{1}{m s^{2}}
$$

where $m$ is the mass of the mover in the translational axes and the moment of inertia in the rotational axes. Since the phase of the transfer function shown in Eq. is -180 degrees, phase has been added with the following lead-lag controller at the desired bandwidth,

$$
C(s)=\frac{X}{F}=K\left(\frac{1+\alpha T s}{1+T s}\right)
$$

where $K$ adjusts the crossover frequency, $\alpha$ adjusts the amount of phase to be added, and $T$ adjusts the frequency at which the phase is added. These values are selected to add 60 degrees of phase at a crossover frequency of $100 \mathrm{~Hz}$, resulting in a bandwidth of approximately $100 \mathrm{~Hz}$. Furthermore, to remove DC error, an integrator $\left(I(s)=1+\omega_{i} / s\right)$ is cascaded with the lead-lag controllers. The gain of the integrator, $\omega_{i}$, is selected to be $10 \mathrm{~Hz}$ so its dynamics do not affect the dynamics of the lead-lag controller. Using the lead-lag controller and integrator, the axes of the rotary table have the following closed loop transfer function:

$$
\frac{X}{X_{R}}=\frac{K \alpha T s^{2}+K\left(1+\omega_{i} \alpha T\right) s+K \omega_{i}}{M T s^{4}+M s^{3}+K \alpha T s^{2}+K\left(1+\omega_{i} \alpha T\right) s+K \omega_{i}}
$$

\section{Simulation and Experimental Results}

Simulations and experiments have been performed to validate the proposed kinematics and control models. The control parameters, mass, and friction of each of the axes are shown in Error! Reference source not found.

A helical tool path has been used to test the machine. The simulated toolpath and corresponding tool position commands are in Figure 2. The experimental tracking errors of all axes are shown in Figure 3 and the accuracy of the machine, as measured by a laser interferometer, is shown in Table 2. The tracking errors of the micromill axes are within $10 \mu \mathrm{m}$. The tracking errors of the rotary table are $200 \mathrm{~nm}$ in translation and $20 \mu \mathrm{rad}$ in rotation, with the exception of the spike in the $\mathrm{C}$-axis tracking error at 2.3 seconds, where a direction change occurs.

The proposed trajectory generation algorithm is used with a feedrate optimization strategy [11] to ensure the planned trajectory does not violate any drive limits. Figure 4 shows the computed jerk of the reference commands. The jerks hit the limits, indicating the algorithm attempts to optimize the feed profile, but does not violate the limits. As it can be seen, the trajectory generation algorithm can be used successfully with existing optimization strategies to generate movement profiles so the 9-axis machine stays within its limits. 
Table 1. Control parameters of the 9-axis machine

\begin{tabular}{|c|c|c|c|c|c|}
\hline \multirow{2}{*}{ Axis } & \multicolumn{3}{|c|}{ PP1 Parameters } & \multirow{2}{*}{ Inertia } & \multirow{2}{*}{$\begin{array}{l}\text { Viscous } \\
\text { Friction }\end{array}$} \\
\hline & $K_{p}$ & $K_{v}$ & $K_{v i}$ & & \\
\hline$x_{c}$ & 275.8 & 0.175 & 61.6 & $21.6 \mathrm{~kg}$ & $272.6 \frac{\mathrm{kg}}{\mathrm{s}}$ \\
\hline$y_{c}$ & 275.3 & 0.624 & 219.0 & $57.8 \mathrm{~kg}$ & $334.9 \frac{\mathrm{kg}}{\mathrm{s}}$ \\
\hline$z_{c}$ & 84.4 & 0.181 & 26.7 & $17.6 \mathrm{~kg}$ & $17.6 \frac{\mathrm{kg}}{\mathrm{s}}$ \\
\hline \multirow{2}{*}{ Axis } & \multicolumn{3}{|c|}{ Lead Lag Parameters } & \multirow{2}{*}{ Inertia } & Viscous \\
\hline & $K$ & \multirow{4}{*}{\multicolumn{2}{|c|}{$\begin{array}{c}\alpha=21.59 \\
T=3.4 \times 10^{-4} \\
\omega_{i}=62.83\end{array}$}} & & Friction \\
\hline$x_{f}, y_{f}, z_{f}$ & 194405 & & & $2.3 \mathrm{~kg}$ & $2.3 \mathrm{~kg}$ \\
\hline$a_{f}, b_{f}$ & 1765 & & & $20900 \mathrm{~kg} \cdot \mathrm{mm}^{2}$ & $0 \frac{\mathrm{kg} \cdot \mathrm{mm}^{2}}{\mathrm{~s}}$ \\
\hline$c_{f}$ & 3424 & & & $40500 \mathrm{~kg} \cdot \mathrm{mm}^{2}$ & $0 \frac{\mathrm{kg} \cdot \mathrm{mm}^{2}}{\mathrm{~s}}$ \\
\hline
\end{tabular}
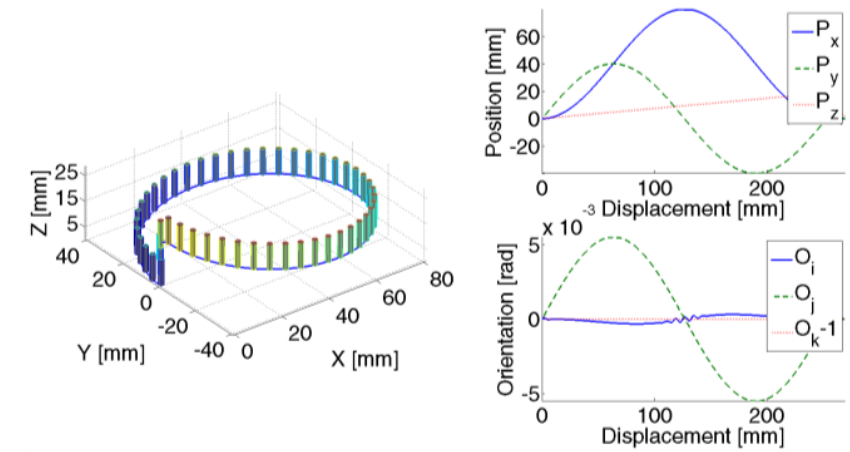

Figure 4. Spatial toolpath and position and orientation commands.
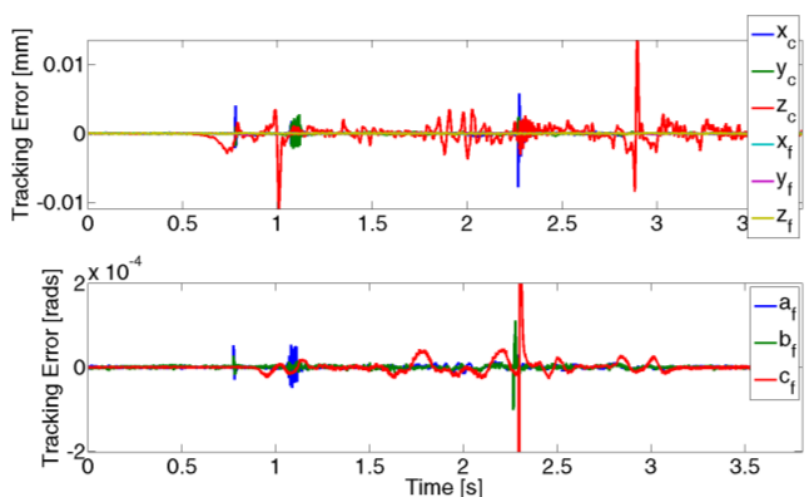

Figure 2. Experimental Tracking error of all axes of the micro-machine

Table 2. Laser accuracy tests for 9-axis machine

\begin{tabular}{|c|c|c|c|}
\hline Micromill & Accuracy & Rotary Table & Accuracy \\
\hline \multirow{2}{*}{$x_{c}$} & \multirow{2}{*}{$3.9 \mu \mathrm{m}$} & $x_{f}$ & $0.6 \mu \mathrm{m}$ \\
\cline { 3 - 4 } & & $y_{f}$ & $0.3 \mu \mathrm{m}$ \\
\hline \multirow{2}{*}{$y_{c}$} & \multirow{2}{*}{$5.1 \mu \mathrm{m}$} & $z_{f}$ & $3.9 \mu \mathrm{m}$ \\
\cline { 3 - 4 } & \multirow{2}{*}{$20.1 \mu \mathrm{m}$} & $a_{f}$ & $0.2 \mathrm{mrad}$ \\
\cline { 3 - 4 }$z_{c}$ & $b_{f}$ & $0.2 \mathrm{mrad}$ \\
\hline
\end{tabular}

\section{Conclusions}

A novel 9-axis micromachining center has been presented. The 9axis micromachining center consists of a micro-mill with three linear, large stroke axes, and a 6 DOF magnetically actuated rotary table with three linear and three rotational degrees of freedom. The fundamental challenge is to deliver a 5 axis tool path by moving all 9 drives with different stroke and acceleration limits simultaneously. The proposed novel geometric trajectory generation algorithm is able to handle the redundant drives without saturating any of the 9 drives. The algorithm optimizes the velocities of redundant drives, i.e. $\mathrm{x}$ axis of the mill with a long stroke $(100 \mathrm{~mm})$ and $\mathrm{x}$ axis of the rotary table
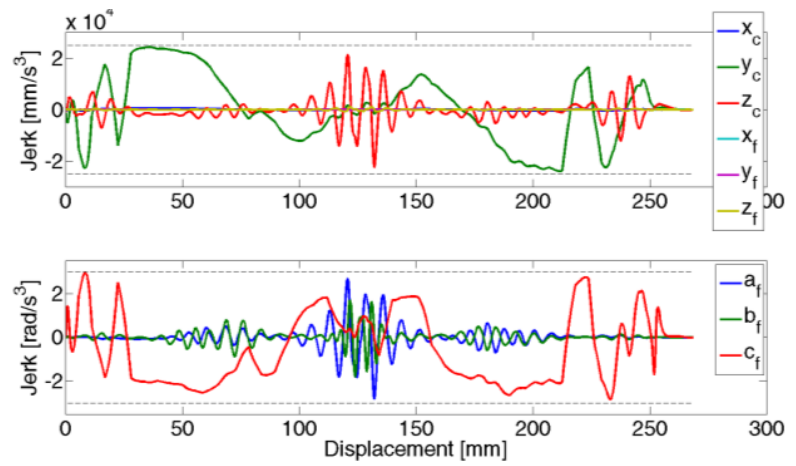

Figure 3. Computed jerks of translational and rotational reference commands

with a short stroke $(0.2 \mathrm{~mm})$, so that both move but positions the tool at the desired location.

The preliminary design, kinematics and control methods allowed the full operation of the novel micro-machine in real time. The researchers are currently investigating new dual-axis control methods with improved bandwidth and trajectory generation, while minimizing the disturbance caused by micro-milling forces. The metrology of the machine will be developed to compensate its volumetric accuracy. The machine will be used in micro-end milling of miniature features and textures.

Acknowledgement: This research has been supported by National Sciences and Engineering Council of Canada, Discovery Grant NSERC 6164-2011

\section{References}

[1] Byrne, G., Dornfeld, D., Denkena, B., 2003, Advancing Cutting Technology, CIRP Annals - Manufacturing Technology, 52:483-507.

[2] Dornfeld, D., Min, S., Takeuchi, Y., 2006, Recent Advances in Mechanical Micromachining, CIRP Annals - Manufacturing Technology, 55:745-768.

[3] Piers, J., 2009, Turboshaft Setup, Internet: http://www.powermems.be/Turboshaft.html, [Mar. 21, 2016]

[4] Halevi, Y., Carpanzano, E., Montalbano, G., Koren, Y., 2011, Minimum energy control of redundant actuation machine tools, CIRP Annals - Manufacturing Technology, 60:433-436.

[5] Takeuchi, Y., Sakaida, Y., Sawada, K., Sata, T., 2000, Development of a 5-Axis Control Ultraprecision Milling Machine for Micromachining Based on Non-Friction Servomechanisms, CIRP Annals - Manufacturing Technology, 49:295-298.

[6] Takeuchi, Y., Yoneyama, Y., Ishida, T., Kawai, T., 2009, 6-Axis control ultraprecision microgrooving on sculptured surfaces with non-rotational cutting tool, CIRP Annals - Manufacturing Technology, 58:53-56.

[7] Lu, X., Usman, I.U.-R., 2012, 6D direct-drive technology for planar motion stages, CIRP Annals - Manufacturing Technology, 61:359-362.

[8] Lu, X., Dyck, M., Altintas, Y., 2015, Magnetically levitated six degree of freedom rotary table, CIRP Annals - Manufacturing Technology, 64:353-356.

[9] Choi, Y.-M., Gweon, D.-G., 2011, A High-Precision Dual-Servo Stage Using Halbach Linear Active Magnetic Bearings, IEEE/ASME Transactions on Mechatronics, 16:925-931.

[10] Erkorkmaz, K., Altintas, Y., 2001, High speed CNC system design. Part I: jerk limited trajectory generation and quintic spline interpolation, International Journal of Machine Tools and Manufacture, 41:1323-1345.

[11] Sencer, B., Altintas, Y., Croft, E., 2008, Feed optimization for five-axis CNC machine tools with drive constraints, International Journal of Machine Tools and Manufacture, 48:733-745.

[12] Dong, J., Ferreira, P.-M., Stori, J.-A., 2007, Feed-rate optimization with jerk constraints for generating minimum-time trajectories, International Journal of Machine Tools and Manufacture, 47:1941-1955.

[13] Yuen, A., Zhang, K., Altintas, Y., 2013, Smooth trajectory generation for five-axis machine tools, International Journal of Machine Tools and Manufacture, 71:11-19.

[14] Whitney, D., 1969, Resolved Motion Rate Control of Manipulators and Human Prostheses, IEEE Transactions on Man-Machine Systems, 10:47-53.

[15] Liegeois, A., 1977, Automatic Supervisory Control of the Configuration and Behavior of Multibody Mechanisms, IEEE Transactions on Systems, Man and Cybernetics, 7:868-871.

[16] Klein, C., Huang, C.-H., 1983, Review of pseudoinverse control for use with kinematically redundant manipulators, IEEE Transactions on Systems, Man and Cybernetics, 13:245-250 
